Voix et Images

volxetimages

\title{
« Être est une activité de fiction. » L'écriture de Suzanne Jacob
}

\section{Christl Verduyn}

Volume 21, numéro 2 (62), hiver 1996

Suzanne Jacob

URI : https://id.erudit.org/iderudit/201234ar

DOI : https://doi.org/10.7202/201234ar

Aller au sommaire du numéro

\section{Éditeur(s)}

Université du Québec à Montréal

\section{ISSN}

0318-9201 (imprimé)

1705-933X (numérique)

Découvrir la revue

\section{Citer cet article}

Verduyn, C. (1996). «Être est une activité de fiction. » L'écriture de Suzanne Jacob. Voix et Images, 21(2), 234-242. https://doi.org/10.7202/201234ar
Résumé de l'article

Résumé

En déclarant qu'*être est une activité de fiction", Suzanne Jacob dévoile les grands points de repère de son écriture. La triade être/agir/écrire constitue en effet un noeud de motifs qui structure son oeuvre, et qui fait l'objet du présent article. Par le biais de la fiction, Jacob témoigne de la nécessité, pour les femmes, d'agir. L'acte d'écrire s'avère extrêmement important, car il permet à la femme d'être présente au monde. 


\title{
«Être est une activité de fiction. » L'écriture de Suzanne Jacob
}

\author{
Christl Verduyn, Université Trent
}

\begin{abstract}
En déclarant qu'être est une activité de fiction., Suzanne Jacob dévoile les grands points de repère de son écriture. La triade être/agir/écrire constitue en effet un noud de motifs qui structure son cuvre, et qui fait l'objet du présent article. Par le biais de la fiction, Jacob témoigne de la nécessité, pour les femmes, d'agir. L'acte d'écrire s'avère extrêmement important, car il permet à la femme d'être présente au monde.
\end{abstract}

Dans un essai publié à l'automne 1988 et intitulé "Conférence-fiction", Suzanne Jacob affirme que "la fiction est la condition de la réalité ${ }^{1}{ }_{n}$. Parmi les fictions, qu'elle définit comme les "structures de perception" $(C F$, p. 85) qui commandent les structures d'organisation, Jacob retient celles qui organisent ce que nous nommons la réalité. Celle-ci n'est qu'une fiction parmi d'autres - une version convenue des choses, une vision du monde. L'art propose d'autres fictions, qui permettent de voir autrement. Ainsi, la littérature fait voir "les espaces du possible, du renouveau, de la mutation, du mouvement, de la régénérescence, c'est-à-dire les espaces de naissance" ( $C F$, p. 87), et affirme " $[\ldots]$ que tout peut être, que tout peut ne pas être, et qu'ÊTRE EST UNE ACTIVITÉ DE FICTION" ( $C F$, p. 87).

Jacob dévoile ainsi les grands points de repère de son ouvre. L'élaboration de l'articulation être/agir/écrire situe son œuvre dans un courant que l'on a qualifié de "métaféministe ${ }^{2}$. Comme beaucoup de ses contemporains-es, l'auteure aborde la question de l'identité humaine (être), ainsi

1. "Conférence-fiction", Possibles, vol. XII, $\mathrm{n}^{\circ}$ 4, automne 1988, p. 85-93. Désormais, les citations tirées de cet essai seront identifiées par le sigle $C F$, suivi du folio.

2. Lori Saint-Martin, "Métaféminisme ", Spirale, octobre 1990, p. 12-13. Par cette notion, on veut signifier que les œuvres métaféministes "absorbent, interrogent [et] font évoluer " le féminisme. "Plus personnelles, plus lisibles, davantage féminines qu'explicitement féministes, explique Lori Saint-Martin, les fictions métaféministes n'annoncent pas la fin du féminisme, mais son évolution, son renouvellement, voire sa pérennité ". 
que le rapport entre la "réalité" et la fiction. Elle souligne l'importance, pour les femmes, d'agir. Elle affirme en particulier la portée - pour elle, et pour les femmes en général - de l'acte d'écrire. En même temps, Jacob cherche à découvrir ce qui peut suivre l'acte d'écriture. Ses protagonistes, inscrites dans l'agir, accomplissent en effet des actes nombreux et variés, multipliant ainsi les liens possibles entre être et fiction chez les femmes. Il est primordial d'étudier le nœud de motifs être/activité/fiction qui structure l'œuvre de Jacob.

Être

Ne pas y être, voilà le sort de la majorité. Voilà ce que chacun tente de surmonter, cette incapacité à y être.

Julie, L'Obéissance ${ }^{3}$

J'écris, affirme Suzanne Jacob, "pour la compréhension de ce je impossible à saisir, toujours en train de se liquéfier, de s'évaporer ${ }^{4}$. Jacob figure parmi les écrivains-es qui interrogent l'expérience de la subjectivité humaine, sujet central dans les courants de pensée modernes, aussi bien féministe que postmoderne. Le concept du je cohérent, centré, logique et autonome que nous a légué la tradition humaniste occidentale a été remis en question. En effet, ce je ne représente pas l'expérience de la subjectivité que connaissaient la majorité des êtres humains. Il fait abstraction de l'influence décisive exercée par des facteurs tels que le sexe, l'appartenance à une classe sociale, les origines ethniques ou raciales, l'orientation sexuelle, et, comme le montre le roman le plus récent de Jacob, l'âge. De nouveaux modes de subjectivité ont été envisagés et ont donné lieu à l'articulation d'un je non centré, multiple, marginal par rapport à la norme. L'éclatement du concept de la subjectivité humaine a permis de découvrir l'expérience de l'altérité, ce qui a ouvert un espace pour la différence, espace marqué, selon plusieurs théoriciens-nes de la modernité ${ }^{5}$, par le féminin.

C'est à partir de ce questionnement que Suzanne Jacob explore la subjectivité. Cette interrogation s'exprime en tout premier lieu par la souffrance, mot récurrent dans son écriture. Selon l'auteure, notre "époque de robot " impose des modèles sociaux violents qui font souffrir ${ }^{6}$, par

3. Suzanne Jacob, L'Obéissance, Paris, Seuil, 1991, p. 27. Désormais, les citations tirées de cet ouvrage seront suivies du sigle $O$ et du folio.

4. Jean Royer, "Comment passer de l'image à l'acte?", Écrivains contemporains. Entretiens 1986-1989, Montréal, l'Hexagone, 1989, p. 143-148.

5. Voir entre autres Alice Jardine, Gynesis: Configurations of Woman and Modernity, Ithaca, Cornell University Press, 1985.

6. Pierrette Roy, "Suzanne Jacob en chanson ou une authenticité retrouvée ", La Tribune, 25 août 1979, p. 33. 
exemple, le couple, la famille, la mère et le besoin de soumission, comme en témoigne L'Obéissance. Le roman entremêle l'histoire de Florence Chaillé, fille d'Yvonne Vézina et mère d'Alice, petite fille précoce qui meurt noyée à l'âge de sept ans, et celle de Marie Cholet, avocate à qui il revient de plaider la cause de Florence, accusée du meurtre de sa fille. Le texte s'ouvre sur la réflexion de Julie. Celle-ci se donne pour tâche de montrer comment des couples qui ont l'air tout à fait ordinaires et qui semblent humains en viennent à perpétrer et à perpétuer des cruautés à l'endroit des jeunes. C'est au sein du couple et à partir de lui que la souffrance se constitue. Pris isolément, les partenaires paraissent plutôt inoffensifs, mais à partir du moment où ils sont ensemble, leur cruauté jaillit. Lorsqu'ils deviennent parents, cette cruauté s'intensifie. Père et mère s'avèrent capables d'actes que l'on pourrait qualifier de criminels. C'est ce que découvre Julie à travers l'enfance de son amie, Marie Cholet. "Le rôle des mères humaines", constate-t-elle, c'est d'installer "de solides interdits par des gifles, des larmes et des cris" ( $O$, p. 48). Quant à l'obéissance, elle est le fruit amer de l'incompréhension. "On se met à obéir à ce qu'on ne comprend pas ", fait remarquer Marie Cholet, alors que ce qu'il faut, c'est comprendre, penser, dire ${ }^{7}$, grandir. C'est le projet qu'entreprennent les protagonistes de Suzanne Jacob: elles refusent les conventions et choisissent la désobéissance plutôt que la fiction dominante de la société.

Quelle est cette fiction pour la femme? Suzanne Jacob la dévoile, pour mieux la rejeter ensuite, à travers son écriture. Elle apparaît chez la petite fille patiente de la première nouvelle de son recueil La Survie ${ }^{8}$ :

Une petite fille patiente qui ne sait pas encore qu'il y a des milliers de petites filles patientes est assise toute droite sur la chaise brune aux barreaux usés de la salle à manger. Elle n'invente rien. Elle ne tourne pas de mots dans sa bouche [...] Elle ne rêve pas [...] Elle ne cherche pas à imaginer [...] Elle n'a pas envie [...] La petite fille patiente est assise dans l'heure de rien. Elle patiente. $(S$, p. 9-10)

Elle apparaît aussi chez la mère dans "Le temps des fraises", femme au visage éteint, indifférente devant la vie en général, et devant le plaisir de manger en particulier ${ }^{9}$. On la voit également chez la protagoniste de la nouvelle "Une femme":

7. Ou écrire, rire, chanter, s'exprimer d'une façon ou d'une autre.

8. Suzanne Jacob, La Survie, Montréal, Le Biocreux, 1979. Les références à ce titre seront indiquées par le sigle $S$, suivi du folio.

9. Chez Suzanne Jacob, le thème de la nourriture est important. Elle s'en sert comme exemple, comme allégorie, comme symbole spirituel. "Les références à la nourriture et à la musculature m'obsèdent parce que je crois que fondamentalement il y a un estomac dans le cerveau et que tout est une question de muscle ... C'est en se nourrissant qu'on participe au règne de la vie et qu'on sort enfin de la définition d'un destin. En fait, je n'ai qu'un seul principe : laissez-moi nourrir mon propre noyau dans le lieu où il est, laissez-moi manger par moi-même. Je n'ai qu'un seul désir, celui que mon désir 
Une femme, debout devant la glace de la cheminée déserte, retire les épingles de sa coiffure [...] Cette femme n'est plongée dans nulle profonde rêverie. Elle n'éprouve aucune nostalgie, son visage n'est ni tendre, ni sévère, ni triste, ni souriant $[\ldots]$ Cette femme est indifférente [...] elle ne ressent rien [...] $(S$, p. 29)

Celle qui attend toujours patiemment, ballottée par le temps qui passe, qui ne rêve pas, n'imagine rien, et n'ose ni inventer ni désirer: voilà celle qui illustre la fiction dominante du côté féminin. C'est une femme aussi aliénée par rapport à elle-même que le je du poème "Accompagnement" de Saint-Denys Garneau ${ }^{10}$. Dans "Une femme", la protagoniste regarde apparaître, dans la glace devant laquelle elle se défait les cheveux, "[...] un visage qui n'est pas le sien, mais qui paraît comme s'il n'avait jamais cessé d'être en même temps que le sien dans la glace, un visage qui semble avoir été là depuis toujours, l'accompagnant" ( $S$, p. 29). L'image de la femme élaborée par la fiction dominante est en effet celle d'un être brisé, voire aliéné, sans émotions fortes, sans désir. Cette image décourageante de la femme que lègue la mère à sa fille ne facilite guère l'être de celle-ci.

Comme d'autres écrivaines contemporaines, Suzanne Jacob attire l'attention sur le personnage de la mère, personnage fortement régi par la fiction dominante. Nicole Brossard a forgé l'expression "mère patriarcale" pour désigner la femme qui se plie à l'image conventionnelle de la mère aux dépens de ses propres désirs de femme ainsi qu'aux dépens de ses filles ${ }^{11}$. Suzanne Jacob examine également ce personnage et ses rapports avec ses filles, notamment dans $L^{\prime} O$ béissance ${ }^{12}$.

Entre Florence Chaillé et sa mère, Yvonne Vézina, ont eu lieu "vingt années d'âpres négociations" ( $O$, p. 39):

Il lui était plus facile [à Yvonne Vézina] de gifler sa fille pour n'importe quelle raison que de s'en prendre à son mari ou à ses fils. Elle pouvait se mettre à hurler après Florence pour qu'elle mette de l'ordre dans sa chambre

m'appartienne enfin. "Nathalie Petrowski, "Suzanne Jacob prend la vie du bon côté", Canadian Composer/Compositeur canadien, $\mathrm{n}^{\circ} 142$, juin 1979, p. 4-7.

10. Saint-Denys Garneau, "Accompagnement", Regards et Jeux dans l'espace, Montréal, Fides, 1972 [1937], p. 97.

11. Voir Nicole Brossard, L'Amèr ou Le Chapitre effrité, Montréal, Quinze, 1977.

12. Dans les premiers ouvrages de l'auteure (exception faite de quelques nouvelles de $\mathrm{La}$ Survie), ces rapports ne figurent pas au premier plan. Ils sont sous-jacents dans La Passion selon Galatée (Paris, Seuil, 1987; les références à ce titre seront indiquées par le sigle PG, suivi du folio), p. 138 et 170 , ainsi que dans Plages du Maine (Outremont, Nouvelle Barre du jour, 1989). C'est pour faire le deuil de leur mère que le frère et la sœur sont allés dans le Maine. Selon les directives de la sœur, les jumeaux s'installent sur la plage devant quatre bouteilles de saké qu'ils doivent vider en regardant la mer. Ce comportement peu typique des vacanciers se maintient jusqu'au jour où la sœur semble avoir assimilé la mort de sa mère. "Notre mère est bien morte!" annonce-t-elle à son frère. "On y va" (p. 45). 
alors que la chambre venait d'être rangée. En cela, Yvonne Vézina n'était pas une femme exceptionnelle, mais une femme ordinaire qui participait simplement à la vitalité de l'espèce humaine, comme c'est le rôle des mères humaines ... (O, p. 48)

Florence adopte un comportement semblable envers sa fille, la petite Alice, pourtant intelligente, pleine de verve et de vigueur:

Dans un premier temps, Florence voulut tempérer les élans de sa fille, mais elle en vint vite à les réprimer, puis à les dompter [...]. Pour lui refroidir les sangs comme elle disait, elle la mit aux douches glacées, puisque les privations de dessert et l'isolement dans la chambre ne suffisaient pas. $(O, \mathrm{p} .71)$

Alice s'adonne à des exercises de perfectionnement de plus en plus complexes qui lui permettent de devenir invisible et d'échapper aux punitions de sa mère.

L'histoire d'Alice n'est pas unique. Elle répète celle de Marie Cholet, avocate renommée, intelligente et belle: "C'était son histoire, cette histoire! La baignoire, tous les samedis. La tête dans l'eau. La terreur." ( $O$, p. 245) La mère de Marie lui tenait la tête dans l'eau pour lui apprendre à ne pas se révolter. Marie se met alors à craindre la mère, "toutes les mères" ( $O$, p. 208). Avoir à défendre Florence Chaillé lui est un supplice qui fait remonter à la surface le souvenir de ses difficiles rapports avec sa mère.

Rapports troubles, donc, entre mères et filles dans l'œuvre de Suzanne Jacob. Mais ces rapports reproduisent ce qui se passe au sein du couple. Si la mère de Marie lui tenait la tête sous l'eau, c'est que son mari frappait les bébés qui pleuraient au lieu de dormir (O, p. 245). Si Florence a demandé à Alice d'entrer dans l'eau de la rivière, c'est que le père d'Alice s'excitait à violer sa femme sous les yeux de sa fille. Rejeter la loi du père n'est pas plus permis que rejeter la loi de la mère. C'est la loi du couple, aveugle devant la souffrance des êtres sans pouvoir. Face à cette réalité, le choix est limité et difficile: traverser les interdits (ce qui peut mener à la mort) ou cultiver l'imaginaire (ce qui peut mener à l'art). Mais avant tout: agir.

Agir

Tout le palais de justice attend le passage à l'acte.

(O, p. 199)

Suzanne Jacob met l'accent sur la nécessité d'agir. L'acte, l'événement et "le fait divers" sont des motifs essentiels de son œuvre. C'est grâce à l'action que les femmes peuvent sortir de la fiction dominante et se libérer des images conventionnelles que leur impose la société. 
Jacob fait ressortir cette idée en puisant dans la mythologie le personnage de Galatée, protagoniste du roman La Passion selon Galatée. Dans la mythologie, Pygmalion tombe amoureux de la statue qu'il a sculptée et demande à Aphrodite de l'animer. La déesse d'amour accepte de souffler la vie à Galatée, qui passe ainsi de l'œuvre d'art à la réalité.

Pour Suzanne Jacob, l'histoire de Galatée soulève plusieurs interrogations ${ }^{13}$. Une fois en vie, Galatée a-t-elle compris le monde immédiatement? Comment a-t-elle fait pour agir? Voilà les questions que pose l'auteure dans La Passion selon Galatée, où elle décrit les efforts de Galatée pour agir. Galatée cherche à s'inventer au moyen d'une suite de gestes et d'événements, elle essaie de discerner "parmi le tumulte des actes qui s'accomplissaient à une vitesse vertigineuse à l'intérieur d'elle-même, l'acte qui la franchissait pour apparaitre aux yeux du monde, dans l'espace ..." $(P G$, p. 232). À l'instar de Galatée, la protagoniste éponyme des Aventures de Pomme Douly ${ }^{14}$ est fascinée par la question du "mécanisme par lequel un seul acte est mis en branle au-dehors pendant que toutes les alternatives à cet acte demeurent présentes à l'esprit " ( $A P D, \mathrm{p} .128)$.

Cette interrogation se poursuit et s'enrichit dans L'Obéissance. Au début du roman, Julie se demande comment générer "concrètement et quotidiennement des actes individuels, des actes personnels" pouvant rompre le pacte de la cruauté et de la manipulation entre êtres humains $(O$, p. 12). Elle-même décide d'agir le jour où une jeune enfant malmenée attire son regard. Dans l'œuvre de Suzanne Jacob, c'est justement la femme qui regarde, qui est témoin, acte privilégié. Le frère de Laura Laur se rappelle que sa sœur " ne parlait pas souvent. Elle regardait $1{ }^{\text {n. L'amant }}$ Gilles constate que Laura, "c'était un regard" ( $L L$, p. 86). Posant les yeux sur les choses, les protagonistes de Suzanne Jacob "voient le monde comme s'il n'avait pas été déjà fabriqué par d'autres et au profit des autres ${ }^{16}$ ". Regarder peut être un acte décisif. Ainsi, une fois qu'elle a vu "des plaies vives, sanglantes et purulentes sur le corps d'une enfant de quatre ou cinq ans" $(O$, p. 17), Julie ne peut pas plaider l'ignorance. Elle décide d'agir. En téléphonant au Service de la protection de l'enfance, elle comprend qu'elle a dû "désobéir à une de [s]es lois internes en [s]'ingérant dans la brûlure de cette enfant strabique" $(O$, p. 26). Car, après sa désobéissance, elle ressent des "vertiges accompagnés parfois de palpitations, de sueurs froides et d'une immense fatigue" ( $O$, p. 29). Mais,

13. Jean Royer, op. cit., 1989. Voir aussi Louise Milot, "Une passion d'auteure ", Lettres québécoises, $n^{\circ} 46$, été 1987 , p. 21-23.

14. Suzanne Jacob, Les Aventures de Pomme Douly, Montréal, Boréal, 1988. Les références à ce titre seront désormais indiquées par le sigle $A P D$, suivi du folio.

15. Suzanne Jacob, Laura Laur, Paris, Seuil, 1983. Les références à ce titre seront indiquées par le sigle $L L$, suivi du folio.

16. Réginald Martel, "Sur le fil très tendu d'une humeur simple ", La Presse, 3 mars 1979, p. D-3. 
comme d'autres personnages féminins de Jacob, Julie sait que "ce qui fait évoluer les choses ce sont les actes délinquants ${ }^{17}$ ".

Suzanne Jacob valorise la délinquance féminine dans la mesure où celle-ci permet aux femmes de se libérer des fictions que l'on a construites à leur sujet. Cette traversée des interdits est un élément fondamental de l'écriture de l'auteure:

J'écris pour en finir avec les interdits et les cloisons. J'ai dû faire un effort pour me décider [à écrire] [...] parce que j'étais obsédée par mes propres interdits [...] [Les traverser] a été une épreuve violente ${ }^{18}[\ldots]$ ça m'a semblé bien long. C'était comme si je transportais un grand sac sur mon dos. Dans le sac, il y avait des idées comme "ne te prends pas pour une autre", "ne parle

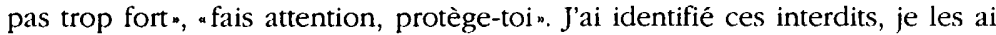
affrontés. Ça n'a pas été facile ${ }^{19}$.

Grâce à leur délinquance, les personnages de Jacob échappent aux conventions de la fiction dominante. "Anti-conformistes, ce sont des anarchistes qui refusent de rester dans leur définition ${ }^{20}$. Ce refus demande une énorme énergie, ce dont les protagonistes de Jacob ne sont pas dépourvues. Pour la plupart passionnées et pleines d'initiatives, elles débordent de vitalité ${ }^{21}$. L'expression "excès jubilatoires ", qu'utilise Julie pour décrire son comportement ( $O$, p. 9), convient à la plupart d'entre elles et correspond à l'expérience de l'auteure. "Les femmes que je connais, moi, elles ont un beau grand respir, elles sont fortes et énergiques. Flore Cocon ${ }^{22}$ a été faite à leur ressemblance. Elle refuse de se laisser enterrer vivante et d'enterrer les autres sous un système d'explication, de justification ${ }^{23}$." Flore Cocon est la première de ces femmes qui s'accordent le droit de désirer, de rêver, d'imaginer, d'inventer, de vivre en dehors du temps ${ }^{24}$, qui décrochent de tout système régissant l'existence humaine. Les personnages féminins refusent la vie programmée, à la différence des personnages masculins qui les entourent. Le frère aîné de Laura Laur, par exemple, ne se sent rassuré que lorsqu'il a un programme à suivre ( $L L$, p. 137). Il ne peut supporter chez sa souur l'absence d'une "ligne directrice" ( $L L$, p. 142). Laura Laur est une "erreur de programma-

17. La Gazette des femmes, vol. V, nº 4, novembre-décembre 1983, p. 25.

18. Conrad Bernier, "Suzanne Jacob: "J'écris pour en finir avec les interdits et les cloisons" "La Presse, 27 mai 1978, p. D-2.

19. Michèle Thibault, "Il faut démolir les murs", Perspectives, 13 octobre 1979, p. 8.

20. Pierre Roberge, "Suzanne Jacob: laisser de l'espace au lecteur pour qu'il puisse imaginer ", La Tribune, 17 décembre 1983, p. E-5.

21. Ce n'est pas le cas de Florence Chaillé de L'Obéissance et des personnages qui lui ressemblent, telle Aglaé, la femme qui partage le compartiment de train avec Marie Cholet lors du "voyage de rêve" de celle-ci.

22. Protagoniste éponyme de Flore Cocon, Montréal, Parti pris, 1978. Les citations de cet ouvrage seront indiquées par le sigle $F C$, suivi du folio.

23. Michèle Thibault, loc. cit., p. 8.

24. Marie Cholet fait un peu exception à ce sujet, du moins à partir de sa rencontre avec Florence Chaillé ( $O$, p. 171-180). 
tion", un personnage qui sort de la trajectoire, qui s'oppose au nivellement. C'est une délinquante, "au sens de ce qui rejette la pensée, le dogme, l'orthodoxie, la science aussi ${ }^{25}$ ". L'auteure explique qu'elle essaie avec Laura Laur "de sortir le mot délinquance de la prison et de l'expérimenter ${ }^{26}$, au point où, plutôt que de renoncer à sa délinquance pour entrer dans la convention, sa protagoniste choisit la mort.

Grâce à leurs trangressions, les personnages féminins de Suzanne Jacob font sortir la "réalité" de la fiction dominante ainsi que du savoir conventionnel, dans la mesure où il fait partie de cette fiction ${ }^{27}$. Julie le constate au début de L'Obéissance: "le savoir continue chaque jour à faire la preuve de son impuissance à contrer chacun des actes misérables perpétrés jour après jour pendant des années" $(O$, p. 11) par les couples, les parents, la société. Par ailleurs, tout le monde sait la même chose. C'est convenu. Voilà pourquoi Laura Laur "était contre savoir une chose ${ }^{28}$. Elle disait qu'une chose sue doit être perdue à jamais" ( $L L$, p. 13). Le refus du savoir traditionnel permet aux personnages féminins d'appréhender la " réalité " selon leurs propres fictions, c'est-à-dire, selon leurs propres structures de perception. Cette transgression marque une étape importante dans l'évolution vers une autre vision du monde. À force d'interroger le savoir conventionnel, les protagonistes de Jacob remettent en question le concept de vérité. Elles affirment le désir d'être vraies, c'est-à-dire de "vouloir exister" ( $L L$, p. 170).

Le désir d'être vraie est d'abord un désir d'être. Pour Laura Laur, comme pour les autres protagonistes de Suzanne Jacob, être est une activité. Il faut passer à l'agir afin de dépasser le statut d'image. Dès lors, chacune peut créer sa propre image, selon ses structures de perception. C'est dans cette activité que sont engagées toutes les protagonistes de Suzanne Jacob. Elles créent partout leurs propres fictions, tout comme l'auteure le fait à travers son écriture.

25. La Gazette des femmes, op. cit., p. 25.

26. Suzanne Jacob, "Pratique d'écriture - notes", Possibles, vol. XI, $\mathrm{n}^{\circ} 3$, printemps/été 1987, p. 186-188.

27. Sous la dictature de la convention de la réalité, les faits sont les faits, et se gardent de l'imaginaire $(C F$, p. 85$)$. Mais les faits sûrs ne trouvent pas leur place dans l'espace de l'imprécision et de l'incertitude qu'habitent les protagonistes de Suzanne Jacob. C'est ce que découvre Marie Cholet, qui avait "l'habitude de [s]'en tenir aux faits, aux événements tels qu'ils apparaissent aux yeux de la loi" $(O$, p. 135). Le procès de Florence Chaillé sèmera le désordre dans la vie bien ordonnée de l'avocate.

28. "À l'école, il fallait suivre. Suivre le programme, pousser, tirer, développer ses os, ses muscles, le poil, devenir quelque chose, quelqu'un, se tenir, se détenir, c'était obligatoire. Laur ne l'a jamais supporté." ( $L L$, p. 15) Laura Laur ressemble à Flore Cocon, qui affirme: "Personne ne me mettra au défi de supporter une vision du monde qui ne me concerne pas." ( $F C$, p. 55) De sa sœur, Jean apprend surtout à "ne pas savoir " $(F C$, p. 13). Serge, le frère aîné, remarque qu'“on ne sait jamais rien quand Laura est là " ( $L L$, p. 143). 


\section{Fiction et écriture}

Écrire est, pour Suzanne Jacob, un acte de connaissance et de naissance. Cet acte révèle que la convention de réalité est une fiction ou une série de fictions régissant les membres d'une société. Grâce à l'écriture, Jacob propose d'autres fictions qui donnent à voir les "espaces de naissance" ( $C F$, p. 87), sources de renouveau, de mutation. "C'est l'écriture qui m'a mise dans le réel", explique Jacob. "C'est l'écriture qui m’arrive":

Écrire a toujours été une façon de résoudre ce qui m'arrivait. C'est réussir à toucher ce qui m'arrive, à le rendre réel [...] Écrire c'est rendre tangibles mes propres événements [...] Si je ne l'écris pas, l'événement ne devient pas réel $^{29}$.

Ainsi, l'écriture engendre le réel. Les mots, observe la protagoniste de $\mathrm{La}$ Passion selon Galatée, "aiguillent les événements": "Si on prononce le mot "citron", on aura aussitôt la bouche inondée de salive: Voilà la preuve", dit Galatée ( $P G$, p. 107). Les mots portent en eux les événements, la matérialité et la présence au monde. Pour Suzanne Jacob, l'écriture, c'est "le corps traversé des mots [...] c'est le lieu de sa présence réelle au monde $30^{\text {}}$. Ce qui la fait aboutir à ce constat:

Je ne suis peut-être pas réelle [...] Finalement, l'écriture devient une forme plus réelle que la mienne. [...] Le texte, lui, est réel. Je ne peux pas répondre du reste ${ }^{31}$ !

Pour Suzanne Jacob, c'est bien l'acte d'écrire, la fiction, qui permet d'être ${ }^{32}$.

29. Jean Royer, op. cit,. 1983.

30. Ibid.

31. Ibid.

32. "C'est extraordinaire comme je me suis sentie vivante et fécondante en écrivant Flore Cocon. Et, d'ailleurs, c'est toujours ce que je ressens profondément quand je chante et quand j'écris. "Conrad Bernier, loc. cit., p. D-2. 